Proceedings

\title{
Inflammation and Chronic Diseases: The Polar Lipid Link ${ }^{\dagger}$
}

\author{
Ronan Lordan ${ }^{1,2}$, Ioannis Zabetakis ${ }^{1,3, *}$ and Alexandros Tsoupras ${ }^{1,3}$ \\ 1 Department of Biological Sciences, University of Limerick, V94 T9PX Limerick, Ireland; \\ Ronan.Lordan@ul.ie (R.L.); Alexandros.Tsoupras@ul.ie (A.T.) \\ 2 Institute for Translational Medicine and Therapeutics, Perelman School of Medicine, \\ University of Pennsylvania, Philadelphia, PA 19104-5158, USA \\ 3 Health Research Institute, University of Limerick, V94 T9PX Limerick, Ireland \\ * Correspondence: Ioannis.Zabetakis@ul.ie \\ + Presented at the 1st International Electronic Conference on Food Science and Functional Foods, \\ 10-25 November 2020; Available online: https://foods_2020.sciforum.net/.
}

Citation: Lordan, R.; Zabetakis, I.; Tsoupras, A. Inflammation and Chronic Diseases: The Polar Lipid Link. 2021, 70, 70 .

https://10.3390/foods_2020-07598

Published: 9 November 2020

Publisher's Note: MDPI stays neutral with regard to jurisdictional claims in published maps and institutional affiliations.

Copyright: $\odot 2020$ by the authors. Licensee MDPI, Basel, Switzerland. This article is an open access article distributed under the terms and conditions of the Creative Commons Attribution (CC BY) license (http://creativecommons.org/licenses/by/4.0/).

\begin{abstract}
Cardiovascular diseases (CVD) are the leading cause of death globally. The focus of scientists and practitioners for decades has been on cholesterol levels and pharmacological ways to control them. Over the past few years, a new school of thought has emerged: inflammation is the underlying cause of chronic non-communicable diseases (NCDs) such as CVD. Therefore, the key to reducing the incidence of chronic diseases is to control the activities of various inflammatory mediators, such as platelet-activating factor (PAF), via diet, exercise, and healthy lifestyle choices. One proposal has been that the polar lipids (PL) present in foods can play a key role via their antithrombotic and anti-inflammatory bioactivities. In this paper, we present our latest views on PAF and how it is related to CVD, and we discuss the role of diet and PL against PAF-induced inflammation.
\end{abstract}

Keywords: inflammation; chronic diseases; NCD; lipids; functional foods; nutraceuticals

\section{Introduction}

Non-communicable diseases (NCD) are a significant burden on health care systems and society. Atherosclerotic cardiovascular diseases (ASCVD or CVD) are one of the main NCDs and are the leading cause of death worldwide. Previously considered a lipid storage disease, CVD is now thought to be far more complex, encompassing a significant proinflammatory state, dysregulated lipid metabolism, and oxidative stress [1]. Over half of the deaths related to cardiometabolic diseases are attributed to maladaptive diet $[2,3]$. Many diets and various dietary constituents have been strongly linked with beneficial effects against CVD [3]. One family of molecules of significant interest for their pro-inflammatory role in CVD and NCD is platelet-activating factor (PAF) and PAF-like lipids. Notably, these lipids have garnered significant attention for their potential modulation by pharmacological and dietary intervention [4,5]. The purpose of this paper is to highlight the role of inflammation in CVD, and considering that diet and lifestyle are modifiable risk factors for CVD prevention, we aim to explore the role that dietary polar lipids (PL) play against inflammation and the onset of CVD via the role of PAF.

\section{Inflammation, Atherosclerosis, and Cardiovascular Diseases}

Systemic inflammation is a biochemical phenomenon involving a plethora of immune cells and pathways as a result of tissue injury or in response to pathogenic insults. During the inflammatory response, our immune system activates various immune cells, 
such as leukocytes, which produce numerous agents that either promote or suppress inflammation. These agents include reactive oxygen species (ROS), cathepsins, elastases, proteinases, and lipid mediators, such as eicosanoids and platelet-activating factor (PAF). If inflammation is left unresolved, it can lead to the oxidative damage of plasma lipoproteins that results in the "inappropriate" recruitment of immune cells to the site of the inflammatory response, thus further exacerbating the response [1].

Atherosclerosis is the underlying cause of most CVD, which are chronic progressive vascular diseases driven by systemic and unresolving inflammation [1,6,7]. Atherosclerosis develops via the induction of endothelial dysfunction and the infiltration of lipids and immune cells into the subendothelium, in which platelets play a significant role [8]. Within the subendothelium, monocytes differentiate into macrophages that go on to engulf oxidized lipids, leading to the formation of foam cells and the characteristic fatty streaks and atherosclerotic plaques. The subsequent fissure, erosion, and potential rupture of the plaque can lead to major cardiovascular events, such as myocardial infarction $[7,9]$. Various lipid mediators play a crucial role in the pro-inflammatory processes that occur in atherosclerosis. Eicosanoids are a group of structurally and stereochemically distinct lipid species that are generated and concentrated by inflammatory cells. Arachidonic acid (AA), which is an $n-6$ 20-carbon polyunsaturated fatty acid (PUFA), and which also happens to be the most prominent PUFA present in cell membranes, is the main substrate for the biosynthesis of eicosanoid molecules implicated in both pro- and anti-inflammatory processes. These eicosanoids include leukotrienes, thromboxanes, prostaglandins, and various other oxidized derivatives [10,11]. However, a less well-researched but important family of lipids that exhibit similar pro-inflammatory activities towards many of the eicosanoids are PAF and PAF-like lipids.

These PAF and PAF-like lipids have been the focus of our research over the last number of years, as they are implicated in various inflammatory conditions. PAF is especially involved in the pro-inflammatory development of atherosclerosis [1,5]. Since its discovery, PAF was recognized as a mediator involved in diverse physiological and pathophysiological processes, such as the mediation of normal inflammatory responses, the regulation of blood circulation and pressure, the regulation of coagulation, glycogen degradation, neurological function, reproduction, fetal implantation, lung maturation, initiation of parturition, and various exocrine gland functions. The classic PAF molecule is characterized by an alkyl ether linkage at the $s n-1$ position, an acetyl group at the $s n-2$ position, and a phosphocholine group at the $s n-3$ position of the glycerol backbone (Figure $1 ; 1-O-$ alkyl-2-sn-acetyl-glycero-3-phosphocholine) [1]. PAF and PAF-like molecules act through their binding to a unique $\mathrm{G}$ protein-coupled receptor known as the PAF-receptor (PAF$\mathrm{R})$. The PAF-R is constitutively expressed on numerous cells, including platelets, leukocytes, and endothelial cells, and is highly expressed by cells within the innate immune and cardiovascular systems [12]. Ligand binding (PAF and/or PAF-like molecules) to the PAF-R subsequently triggers multiple intracellular signaling pathways, depending on the target cell and PAF concentration in blood or tissue. If unregulated, these signaling pathways eventually lead to a pro-inflammatory state, oxidative stress, and increased platelet and leukocyte reactivity, which can lead to endothelial dysfunction and the eventual development of atherosclerotic plaques [1].

However, PAF has an Achilles heel in that several compounds of pharmacological and natural origin seem to be able to dampen or inhibit the binding of PAF and PAF-like lipids by interacting with or blocking the PAF-R. Indeed, polar lipids (PL) are one such series of phospholipids and sphingolipids that have structural similarities to PAF and PAF-like lipids which have been the subject of intensive research due to their anti-PAF effects. 


\section{The Potential Use of Platelet-Activating Factor Inhibitors as Therapeutics and Preventatives of Disease}

Research into potential physiological and therapeutic ways of suppressing PAF activity has demonstrated that endogenous or ingested PAF inhibitors could inhibit the actions of PAF [5]. This has led to the expansion of the study of PAF inhibitors of natural or synthetic origin. PAF inhibitors can be classified by their various chemical structures, and they can be classified by their interaction with the PAF-R, e.g., specific and non-specific inhibitors $[1,5]$. Non-specific PAF inhibitors are compounds that inhibit certain processes in the PAF-induced signal transduction pathways, such as calcium channel blockers, G protein inhibitors, intracellular calcium chelators, etc. On the other hand, specific PAF inhibitors competitively or noncompetitively bind with the PAF-R, e.g., PL and PAF-like lipids. In terms of their structures, PAF inhibitors can be PAF analogues such as PL, or they can have different structures, as many are phenolics, dihydropyridines, nitrogen heterocyclic compounds, and various other natural medicinal or synthetic compounds $[1,5,13,14]$. Many of these compounds are associated with healthy diets, which have been shown to be beneficial for the primordial and primary prevention of CVD. Therefore, dietary PAF inhibitors such as dietary PL are of significant interest.

\section{PAF Inhibitors and Anti-Inflammatory Foods}

As eluded to previously, there are several modifiable CVD risk factors that may reduce systemic inflammation. These include the adoption of a healthy diet and lifestyle $[3,7,15,16]$. As demonstrated by the studies of the DASH diet (dietary approaches to stop hypertension) and the Mediterranean diet, a person's diet can directly affect CVD risk factors and mortality [3,17-19]. Consequently, many food constituents of various healthy diets have been investigated to understand how these foods, or combinations of them, can reduce the risk of CVD. This has led to expansive research on food and nutrition. While previous research has suggested that dietary fat, particularly from foods high in saturated fatty acids (SFA), may contribute to CVD, it turns out that dietary fats may actually be beneficial, depending on their sources. Indeed, even SFA in large studies have been shown to be potentially beneficial against stroke, although more research is required [20]. In any case, it generally seems that individual lipid species all have their own potential pro-inflammatory, anti-inflammatory or neutral effects on health.

Many foods and microconstituents of the cardioprotective Mediterranean diet have been associated with anti-inflammatory and protective effects against ASCVD, none more famous than olive oil [17]. Among those many beneficial phenolic compounds and monounsaturated fatty acids (MUFA) are the PL, which have exhibited anti-inflammatory and antithrombotic biological activity against PAF-induced effects in vitro and in vivo [14]. Indeed, fish, dairy, wine, meat, and various other foods of the Mediterranean diet also possess PL with anti-PAF properties that may be beneficial against ASCVD [14]. The current theory is that these PL are PAF analogues that can reduce the pro-inflammatory capacities of PAF (Figure 1). However, there is also evidence that PL bearing bioactive $n-3$ fatty acids may also act as a vehicle to deliver these anti-inflammatory lipids to various tissues, thereby altering the cellular membrane by reducing the levels of pro-inflammatory lipids such as AA [21]. Indeed, this may explain why there is so much heterogeneity across various studies investigating the utility of omega-3 $(n-3)$ fatty acids for treating CVD, as these fatty acids are usually administered in these trials in their neutral forms (esters, triglycerides, free fatty acids), thus leading to various effects. However, the food and meals that we consume are complex and full of both beneficial and detrimental microconstituents with implications for our cardiovascular health. It is imperative that further research is conducted to discern the magnitude of the cardioprotective benefit obtained from dietary PL against the pro-inflammatory effects of PAF in human studies. 

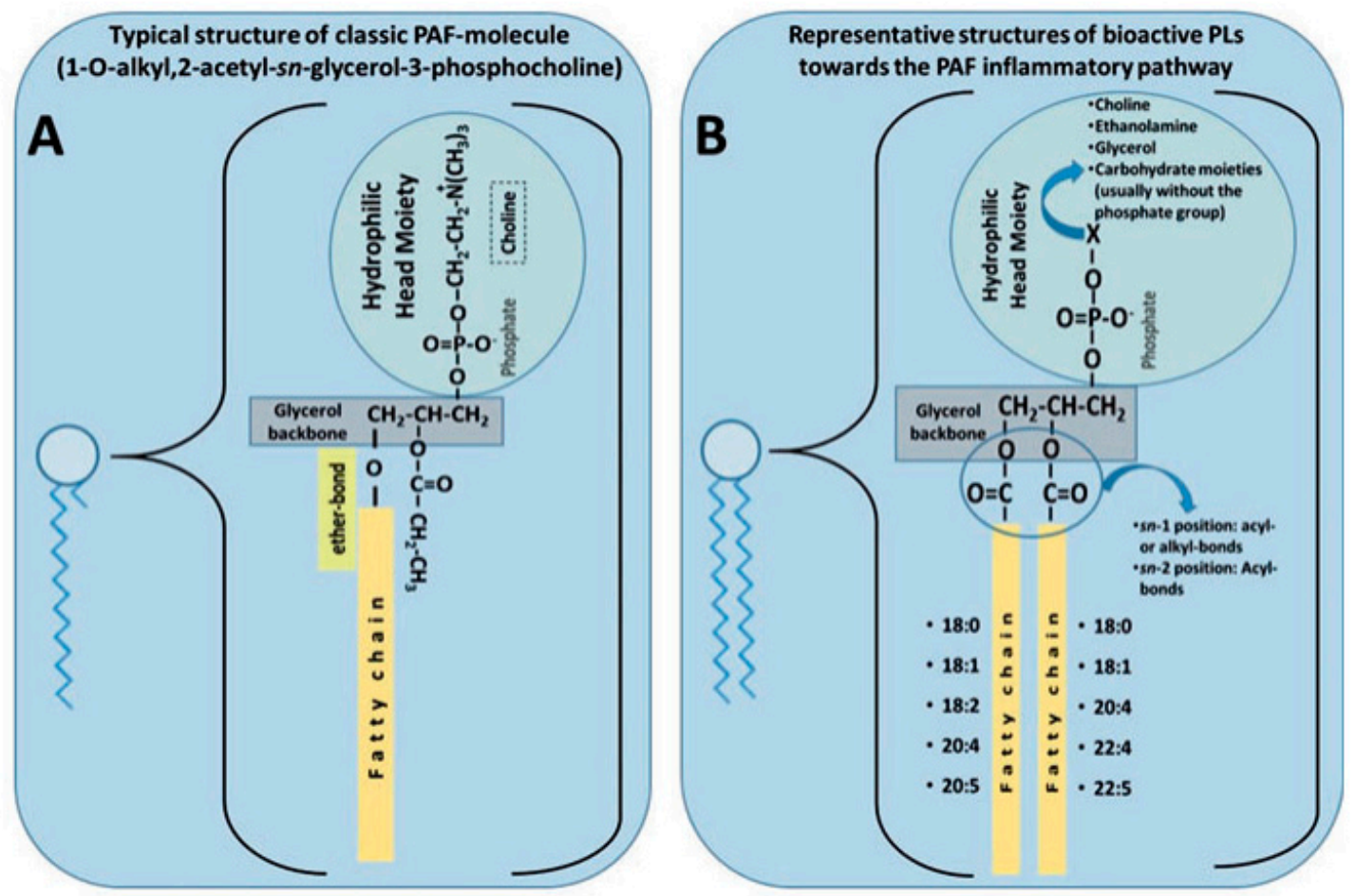

Figure 1. (A) The characteristic structure of platelet-activating factor (PAF). (B) A representative illustration of the general structure of bioactive polar lipids (PL). Reproduced with permission [1].

\section{Conclusions}

The aim of this paper is to present how PAF is related to CVD and how diet may modulate the activities of PAF to reduce the incidence or burden of CVD. It is clear that while there is a lot of promise that PL may exhibit cardioprotective effects versus the PAF pathway, this has yet to be fully elucidated. Large-scale trials are required to fully understand the mechanisms governed by PAF, and its role in relation to chronic inflammation and CVD.

Author Contributions: All authors contributed equally to this work.

Conflicts of Interest: No conflict of interest to declare.

\section{References}

1. Tsoupras, A.; Lordan, R.; Zabetakis, I. Inflammation, not cholesterol, is a cause of chronic disease. Nutrients 2018, 10, 604.

2. Billingsley, H.E.; Carbone, S.; Lavie, C.J. Dietary fats and chronic noncommunicable diseases. Nutrients 2018, 10, 1385.

3. Yu, E.; Malik, V.S.; Hu, F.B. Cardiovascular disease prevention by diet modification: JACC health promotion series. J. Am. Coll. Cardiol. 2018, 72, 914-926.

4. Lordan, R.; Tsoupras, A.; Zabetakis, I. The potential role of dietary platelet-activating factor inhibitors in cancer prevention and treatment. Adv. Nutr. 2019, 10, 148-164.

5. Lordan, R.; Tsoupras, A.; Zabetakis, I.; Demopoulos, A.C. Forty years since the structural elucidation of platelet-activating factor (PAF): Historical, current, and future research perspectives. Molecules 2019, 24, 4414.

6. Libby, P. Inflammation in atherosclerosis. Nature 2002, 420, 868-874.

7. Lordan, R.; Zabetakis, I. Invited review: The anti-inflammatory properties of dairy lipids. J. Dairy Sci. 2017, 100, 4197-4212.

8. Lordan, R.; Tsoupras, A.; Zabetakis, I. Platelet activation and prothrombotic mediators at the nexus of inflammation and atherosclerosis: Potential role of antiplatelet agents. Blood Rev. 2020, 100694, doi:10.1016/j.blre.2020.100694.

9. Bäck, M.; Yurdagul, A.; Tabas, I.; Öörni, K.; Kovanen, P.T. Inflammation and its resolution in atherosclerosis: Mediators and therapeutic opportunities. Nat. Rev. Cardiol. 2019, 16, 389-406.

10. Calder, P.C. The use of n-3 polyunsaturated fatty acids as therapeutic agents for inflammatory diseases. Immunol. Endocr. Metab. Agents 2009, 9, 45-54. 
11. Calder, P.C. Omega-3 polyunsaturated fatty acids and inflammatory processes: Nutrition or pharmacology? Br. J. Clin. Pharmacol. 2013, 75, 645-662.

12. Montrucchio, G.; Alloatti, G.; Camussi, G. Role of platelet-activating factor in cardiovascular pathophysiology. Physiol. Rev. 2000, 80, 1669-1699.

13. Sioriki, E.; Lordan, R.; Nahra, F.; van hecke, K.; Zabetakis, I.; Nolan, S.P. In vitro anti-atherogenic properties of n-heterocyclic carbene aurate(I) compounds. ChemMedChem 2018, 13, 2484-2487.

14. Lordan, R.; Nasopoulou, C.; Tsoupras, A.; Zabetakis, I. The anti-inflammatory properties of food polar lipids. In Bioactive Molecules in Food; Mérillon, J.M., Ramawat, K.G., Eds.; Springer International Publishing: Cham, Switzerland, 2018; pp. 1-34.

15. Masana, L.; Ros, E.; Sudano, I.; Angoulvant, D. Is there a role for lifestyle changes in cardiovascular prevention? What, when and how? Atheroscler. Supp. 2017, 26, 2-15.

16. O'Keefe, J.H.; Gheewala, N.M.; O'Keefe, J.O. Dietary strategies for improving post-prandial glucose, lipids, inflammation, and cardiovascular health. J. Am. Coll. Cardiol. 2008, 51, 249-255.

17. Martínez-González, M.A.; Gea, A.; Ruiz-Canela, M. The mediterranean diet and cardiovascular health. Circ. Res. 2019, 124, 779798.

18. Soltani, S.; Chitsazi, M.J.; Salehi-Abargouei, A. The effect of dietary approaches to stop hypertension (DASH) on serum inflammatory markers: A systematic review and meta-analysis of randomized trials. Clin. Nutr. 2018, 37, 542-550.

19. Tierney, A.; Lordan, R.; Tsoupras, A.; Zabetakis, I. Chapter 8-Diet and cardiovascular disease: The mediterranean diet. In The Impact of Nutrition and Statins on Cardiovascular Diseases; Zabetakis, I., Lordan, R., Tsoupras, A., Eds.; Elsevier: Cambridge, MA, USA, 2019; pp. 267-288.

20. Dehghan, M.; Mente, A.; Zhang, X.; Swaminathan, S.; Li, W.; Mohan, V.; Iqbal, R.; Kumar, R.; Wentzel-Viljoen, E.; Rosengren, A.; et al. Associations of fats and carbohydrate intake with cardiovascular disease and mortality in 18 countries from five continents (PURE): A prospective cohort study. Lancet 2017, 390, 2050-2062.

21. Lordan, R.; Redfern, S.; Tsoupras, A.; Zabetakis, I. Inflammation and cardiovascular disease: Are marine phospholipids the answer? Food Funct. 2020, 11, 2861-2885. 